\title{
Effect of resource supply on interactions between foliar endophytic and root mycorrhizal fungi in perennial ryegrass
}

\author{
Q. LIU ${ }^{1}$, A. J. PARSONS ${ }^{1}$, H. XUE ${ }^{1}$, J. A. NEWMAN ${ }^{2}$ and S. RASMUSSEN ${ }^{1}$ \\ ${ }^{1}$ AgResearch, P.B. 11008, Palmerston North \\ ${ }^{2}$ Dept. Environmental Biology, University of Guelph, Ontario, Canada N1G2W1
}

Susanne.rasmussen@agresearch.co.nz

\begin{abstract}
Both foliar endophytes and root arbuscular mycorrhizal fungi can infect perennial ryegrass and improve its performance, mainly by improving herbivore resistance and phosphorus (P) uptake, respectively. Here, we report a study of how the endophytic fungus (Neotyphodium lolii) and two mycorrhizal fungi (Glomus spp.) are affected by $\mathrm{P}$ supply and perennial ryegrass (Lolium perenne) cultivars differing in water soluble carbohydrate content. Endophyte and alkaloid concentrations were reduced at high $\mathrm{P}$ supply and in the high sugar grass (HSG) 'AberDart', and these effects were modulated by the presence of mycorrhizal fungi. High P supply resulted also in a strong reduction of mycorrhizal colonisation, while the effect of the high sugar grass was subtle and modulated by endophyte infection. Our results show that the simultaneous association of perennial ryegrass with both endophytic and mycorrhizal endosymbionts can negatively affect each endosymbiont, but this depends on other factors such as resource supply and genetic background of the partners.
\end{abstract}

Keywords: ryegrass, phosphate, high sugar grass, endophyte, mycorrhizae, alkaloids

\section{Introduction}

Field grown perennial ryegrass plants are often infected with endophytic Neotyphodium spp. and root specific arbuscular mycorrhizal Glomus spp. fungi. Both endosymbionts can confer benefits to temperate grasses, mainly by improving herbivore resistance and phosphorus (P) uptake, respectively. Studies on the interactions of host plants with either one of these fungal symbionts are numerous, but studies considering tripartite interactions between endophyte and mycorrhizal fungi and host plants are rare. It has been shown that endophytic fungi can suppress mycorrhizal colonisation and fungal spore germination (Müller 2003; Omacini et al. 2006; Antunes et al. 2008). Effects of mycorrhizal colonisation on endophyte infection have been much less studied, although it has been reported that mycorrhizal infection can reduce the level of endophyte-conferred herbivore resistance (Vicari et al. 2002). Alkaloid concentrations in the grass-endophyte symbiosis vary considerably depending on season, weather and nutrient supply, and there is conflicting evidence of the effects of nutrients on alkaloid production (Malinowski \& Belesky 2000; Rasmussen et al. 2007).

In a previous study we have shown that both endophyte and alkaloid concentrations were reduced in perennial ryegrass at high nitrogen $(\mathrm{N})$ and in the HSG 'AberDove'(Rasmussen et al. 2007), demonstrating that simple changes in nutrient supply and resource (energy) related plant traits can affect the symbiotic association between ryegrass and its fungal endophytes. Here, we tested if P supply had a similar effect on endophytes and if interactions between mycorrhizal Glomus fungi and foliar endophytes are affected by nutrient and resource supply in a different HSG 'AberDart'.

\section{Methods}

We used two Lolium perenne cultivars, a HSG 'AberDart' and a control 'Fennema' that differ inherently in water soluble carbohydrate contents. Seedlings were artificially inoculated by two N. lolii endophyte strains, Common Strain Lp19 (CS) or AR1; and/or by two mycorrhizal fungi, G. mosseae (GM) or G. intraradices (GI). Non-inoculated endophytefree (EF) and/or mycorrhizal-free (MF) plants were used as controls. A total of 360 plants were grown in sand media and kept in controlled climate chambers for 12 weeks $\left(14 \mathrm{~h} \mathrm{light,} 20^{\circ} \mathrm{C} / 10 \mathrm{~h}\right.$ dark, $10^{\circ} \mathrm{C}$ ). Halfstrength Hoagland nutrient solution containing either $0.05 \mathrm{mM}$ (low P) or $2 \mathrm{mM}$ (high P) $\mathrm{KH}_{2} \mathrm{PO}_{4}$ was added weekly. During the whole growth period plants were watered every second day to "pot field capacity". Plants were trimmed every 4 weeks to $6 \mathrm{~cm}$ above-ground, trimmings were oven-dried and dry weights recorded. At the final harvest leaf blades were frozen and ground in liquid nitrogen and then freeze-dried. Endophyte concentrations were estimated based on quantitative polymerase chain reaction (qPCR) of genomic DNA ( $g$ DNA) isolated from infected plant tissues (Rasmussen et al. 2007). Colonisation of roots with mycorrhizae was microscopically assessed. Peramine and water soluble carbohydrates were extracted and quantified as described previously (Rasmussen et al. 2007).

The overall experiment was a split-unit design, with 
two cultivars, three levels of foliar endophytes, and three levels of mycorrhizal fungi as the whole unit treatment $(2 \times 3 \times 3 \times 10$ replicates $)$ with two levels of $\mathrm{P}$ supply as the sub-unit treatment (genotypes across $\mathrm{P}$ treatments being identical). All analyses were conducted using JMP statistical software version 7.0. Box-Cox transformation was used to homogenise the error variances for statistical analysis and report, uniformly, the untransformed means and standard error of the means. Tukey's Honestly Significant Difference (HSD) test was used to assist in the interpretation of significant effects and interactions.

\section{Results}

In leaf blades, endophyte concentrations were significantly reduced at high $\mathrm{P}$ compared with low $\mathrm{P}$ supply $(\mathrm{P}<0.001)$; the reduction was stronger in the HSG

Figure 1 Concentrations of endophyte (a) and (b) and peramine (c), (d) and (e) in leaf blades. Endophyte concentrations were assessed by qPCR of an endophyte specific gene ( $N$. Iolii chitinase $A$; $N / C h i A$ ) in total (plant and fungal) gDNA isolated from the individual tissues. $F=$ 'Fennema' cultivar; $A=$ 'AberDart' cultivar; MF = mycorrhizal-free; $\mathrm{GM}=\mathrm{G}$. mosseae infected; $\mathrm{Gl}=\mathrm{G}$. intraradices infected; CS = endophyte common strain infected; AR1 = endophyte AR1 strain infected. Different letters denote means that are significantly different according to Tukey's HSD test. Error bars represent standard error (SE).
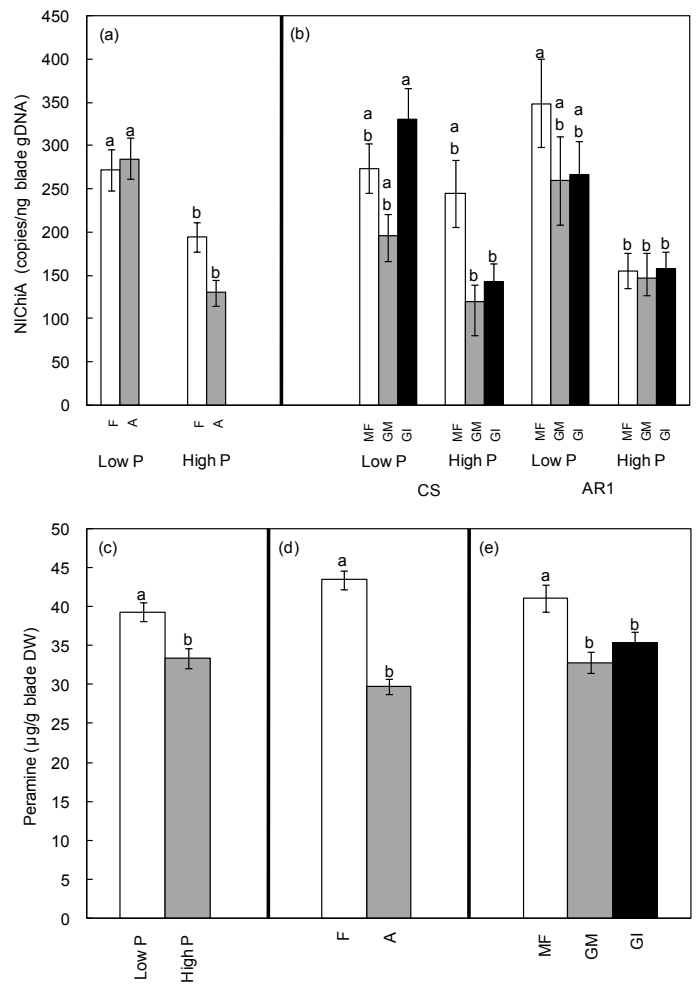

'AberDart' than in the control grass 'Fennema' (Fig. 1a). Mycorrhizal inoculation per se did not affect endophyte concentration; however, it did modulate the response of endophyte to $\mathrm{P}$ in a complex three-way interaction $(\mathrm{P}<0.05)$, which also depended on the endophytic strain (Fig. 1b). In CS infected plants, high P supply reduced endophyte concentrations by more than $50 \%$, but only if plants had been inoculated with $G$. intraradices. In AR1 infected plants, the effect of $P$ was strongest in mycorrhizal-free plants, also resulting in a more than $50 \%$ reduction of endophyte concentrations at high $\mathrm{P}$ compared to low $\mathrm{P}$ supply. Peramine concentrations in leaf blades were significantly reduced at high $\mathrm{P}$ (Fig. 1c; $\mathrm{P}<0.001$ ), in the HSG 'AberDart' (Fig. 1d; $\mathrm{P}<0.001$ ), and in plants colonised with both mycorrhizal species (Fig. 1e; $\mathrm{P}<0.001$ ); none of the interactions between above factors were significant.

Mycorrhizal colonisation rates in plants grown at high $\mathrm{P}$ supply were low (means $\pm \mathrm{SE}$ : low $\mathrm{P}=9.09 \% \pm$ 0.92 ; high $\mathrm{P}=0.41 \% \pm 0.14$ ); we therefore statistically analysed only the data sets from plants grown at low P. At low $\mathrm{P}$ supply, mycorrhizal colonisation rates were affected by a three-way cultivar $\mathrm{x}$ mycorrhizae $\mathrm{x}$ endophyte interaction $(\mathrm{P}<0.05)$. Colonisation rates of $G$. mosseae were not significantly affected by endophyte infection or grass cultivar (Fig. 2). However, colonisation rates of $G$. intraradices were significantly reduced in endophyte infected 'Fennema' plants, but not in 'AberDart'.

High molecular weight water soluble carbohydrates (HMW WSC) were significantly higher in leaf blades of the HSG 'AberDart' compared with the control cultivar 'Fennema' (Fig. 3a; $\mathrm{P}<0.001$ ). Even though the interaction between $\mathrm{P}$ supply and mycorrhizal inoculation was significant $(\mathrm{P}<0.05)$, Tukey's HSD test was unable to differentiate between the different treatment combinations (Fig. 3b). Low molecular weight water soluble carbohydrates (LMW WSC) were not different between the two cultivars, but the observed increase of LMW WSC levels at high $\mathrm{P}$ compared with low $\mathrm{P}$ was significant only in 'Fennema' (Fig. $3 c ; \mathrm{P}<0.001)$. HMW WSCs in roots were increased at high $\mathrm{P}$ compared with low $\mathrm{P}$ supply in both cultivars, and this effect was stronger in 'Fennema', resulting in significantly higher levels of HMW WSCs in roots of 'Fennema' compared with the HSG 'AberDart' at high $\mathrm{P}$ (Fig. 3d; $\mathrm{P}<0.001$ ). $\mathrm{P}$ supply also interacted with mycorrhizal inoculation $(\mathrm{P}<0.01)$, but the interaction was too subtle for Tukey's HSD test to distinguish between treatment combinations (Fig. 3e). LMW WSC concentrations in roots were higher at high $\mathrm{P}$ compared to low $\mathrm{P}$ supply (Fig. 3f; $\mathrm{P}<0.001$ ); none of the other main effects or interactions were significant. 
Figure 2 Arbuscular mycorrhizal fungal (AMF) colonisation in roots of perennial ryegrass plants grown at low $\mathrm{P}$ supply. $\mathrm{EF}=$ endophyte-free; $\mathrm{CS}=$ endophyte common strain infected; AR1 = endophyte AR1 strain infected; $\mathrm{GM}=\mathrm{G}$. mosseae infected; $\mathrm{Gl}=$ $G$. intraradices infected. Different letters denote means that are significantly different according to Tukey's HSD test. Error bars represent standard error (SE).

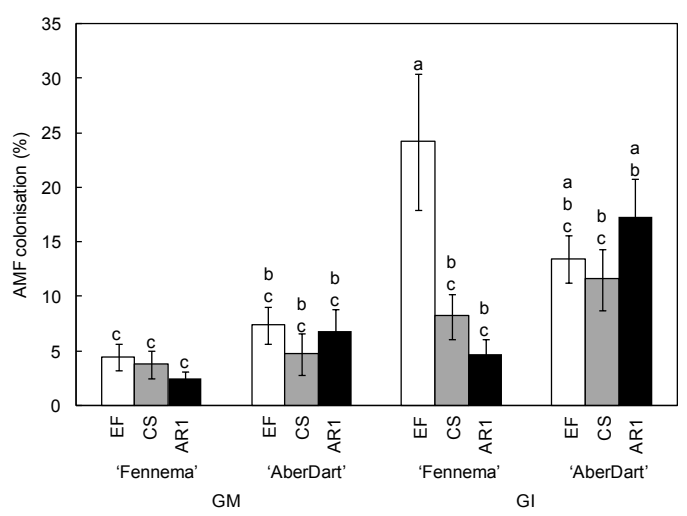

\section{Discussion}

\section{Effects of $P$ supply on foliar endophytes and mycorrhizal fungi}

In the present study high P supply had a strong negative effect on foliar endophyte and alkaloid concentrations in infected leaf blades of perennial ryegrass plants. This effect of high $\mathrm{P}$ supply, in reducing endophyte and alkaloids concentrations, is similar to what has been seen for high $\mathrm{N}$ supply in our previous study. There we hypothesised that high resource supply might stimulate plant shoot growth more than endophyte growth, thereby 'diluting' endophyte and alkaloid concentrations (Rasmussen et al. 2007). High P supply also strongly depressed mycorrhizal colonisation, consistent with previous studies on other plants (Smith $\&$ Read 1997), and it has been shown that signalling molecules such as strigolactones, which are exuded by P starved plants only, mediate this mycorrhizal response (Yoneyama et al. 2007).

\section{Effects of carbohydrate content on foliar endophytes and mycorrhizal fungi}

High sugar grasses (HSG) have been bred for accumulation of high fructan levels in leaf blades, and here we show that 'AberDart' did accumulate higher levels of fructans compared to 'Fennema', consistent with previous studies (Parsons et al. 2004). Endophyte and alkaloid concentrations were reduced in the HSG 'AberDart' compared with the control cultivar 'Fennema', also consistent with a previous study using the HSG 'AberDove' (Rasmussen et al. 2007; 2008a). However, we also note here that the negative effect of the HSG 'AberDart' on endophyte concentrations was
Figure 3 Concentrations of high molecular weight (HMW) and low molecular weight (LMW) water soluble carbohydrates (WSCs) in leaf blades (a), (b) and (c) and roots (d), (e) and (f). F = 'Fennema' cultivar; $A=$ 'AberDart' cultivar; MF = mycorrhizal-free; $\mathrm{GM}=\mathrm{G}$. mosseae infected; $\mathrm{Gl}=\mathrm{G}$. intraradices infected; $C S$ = endophyte common strain infected; AR1 = endophyte AR1 strain infected. Different letters denote means that are significantly different according to Tukey's HSD test. Error bars represent standard error (SE).
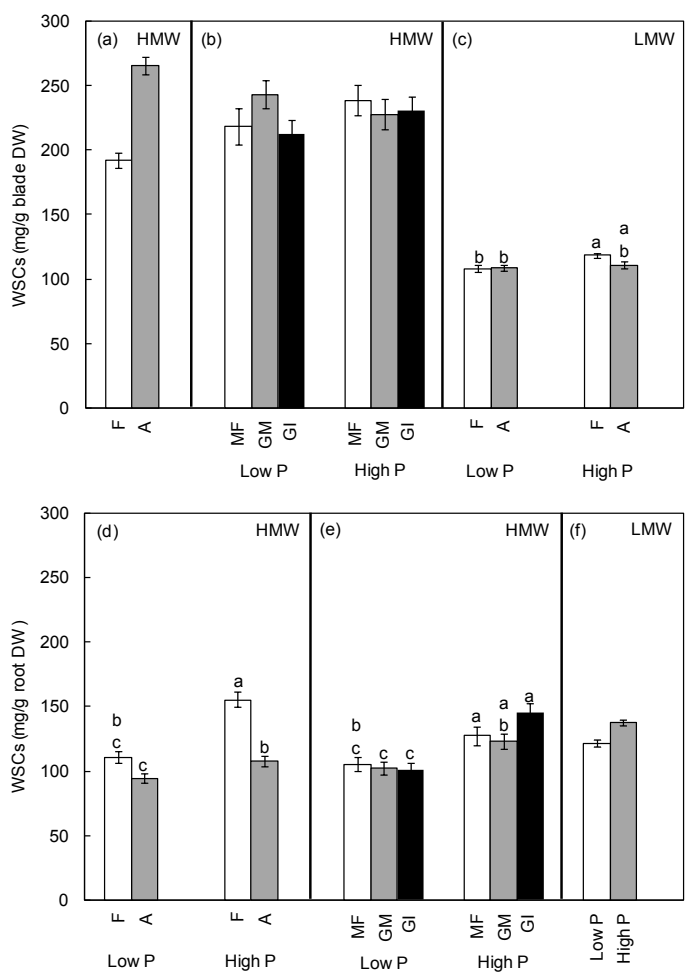

modulated by $\mathrm{P}$ supply and mycorrhizal inoculation; endophyte concentrations were different between the two cultivars only at high P supply, clearly showing that the interaction between high sugar grasses and endophytes can be affected by other factors such as nutrient supply or the presence of mycorrhizal fungi.

In roots, water soluble carbohydrate (WSC) concentrations were the same in the two cultivars at low P supply. At high P supply, high molecular weight WSCs were lower in the HSG 'AberDart' compared with 'Fennema', possibly indicating a difference in sugar translocation in HSG compared with other ryegrass cultivars. Earlier we reported that endophyte infection increased WSC concentrations in the control sugar grass 'Fennema', but not in the HSG 'AberDart' (Rasmussen et al. 2007; 2008a). We did not see an effect of endophyte infection on WSC levels in the present study, but concentrations were much higher compared with our previous study (approx. 350 and 
$150 \mathrm{mg} / \mathrm{g}$ leaf blade DW, respectively), probably due to longer regrowth periods after cutting (4 and 2 weeks, respectively). This demonstrates that any effect endophyte infection might have on host plant water soluble carbohydrates depends on other factors affecting water soluble carbohydrate concentrations, such as harvesting time and cultivar. Mycorrhizal colonisation was not differentially affected in the two cultivars.

\section{Interactions between mycorrhizal fungi and foliar endophytes}

In this study, we hypothesised that foliar endophyte and root mycorrhizal fungi interact with each other if simultaneously present in perennial ryegrass plants, and that the interactions between them are affected by resource supply. Our results show that the effects of mycorrhizal inoculation on endophyte and peramine concentrations interacted with $\mathrm{P}$ supply, cultivar, and endophytic strain. In leaf blades, endophyte concentrations were reduced by mycorrhizal inoculation, but only at high P supply and in CS infected plants, but not at low P supply or in AR1 infected plants. This indicates that studies on the effects of mycorrhizal colonisation need to control for factors like mycorrhizal species, resource supply, genetic background of plants and even foliar endophytic strains, if the outcomes of mycorrhizal infection are to be compared or generalised across a range of ecological studies.

The effect of endophyte infection on mycorrhizal colonisation was subtle and also depended on other factors; specifically mycorrhizal colonisation in $G$. intraradices inoculated 'Fennema' plants. A reduction in mycorrhizal colonisation has previously been reported for several endophyte-infected Lolium species (Müller 2003; Omacini et al. 2006) and it has been suggested that endophytic alkaloids directly transferred to the roots or via leachates from endophyte-infected grass litter might inhibit mycorrhizal fungi (Antunes et al. 2008). We show here that mycorrhizal colonisation was reduced in both CS (produces peramine, lolitrem B, and ergovaline) and AR1 (does not produce lolitrem B or ergovaline) $N$. lolii infected 'Fennema'. This makes it unlikely that the antagonistic effects on mycorrhizal fungi are caused by the endophytic alkaloids lolitrem $\mathrm{B}$ and ergovaline, but our results do not rule out that peramine negatively affected mycorrhizal colonisation. However, we have demonstrated in previous metabolomics studies of the $N$. lolii - perennial ryegrass association (Rasmussen et al. 2008a, b) that a wide range of other, non-alkaloid metabolites are affected in endophyte-infected tissues, which could also be involved in the negative effects of foliar-based endophytic fungi on the root-based mycorrhizal fungi seen in this and other studies.

Our results show that the simultaneous association of temperate grasses with both foliar and root symbiotic fungi can result in the reduction of each endosymbiont, but this depends on other factors such as resource supply or grass cultivar. As this could have both negative (e.g. from reduced endophytic anti-herbivorous alkaloids and/or mycorrhizal $\mathrm{P}$ supply) and positive effects (e.g. from reduced nutrient requirements of the endosymbionts from the host plant), tripartite associations may shift the balance of mutualismparasitism between a host plant and its symbionts, and the degree of this shift depends on a range of other factors, like nutrient supply and genetic background of host plants, mycorrhizal and endophytic fungi.

\section{ACKNOWLEDGEMENTS}

This work has been partially funded by the NZ Foundation of Research, Science and Technology (contracts AGRX0501, C10X0203, PROJ 10333-ECOS-AGR) and AGMARDT NZ (postdoctoral fellowship), and was conducted at AgResearch Grasslands, New Zealand. We thank Dr. Geoffrey A. Lane (AgResearch) for providing us with a mass spectrometry method for the analysis of peramine.

\section{REFERENCES}

Antunes, P.M.; Miller, J.; Carvalho, L.M.; Klironomos, J.N.; Newman, J.A. 2008. Even after death the endophytic fungus of Schedonorus phoenix reduces the arbuscular mycorrhizas of other plants. Functional Ecology 22: 912-918.

Malinowski, D.P.; Belesky, D.P. 2000. Adaptations of endophyte-infected cool season grasses to environmental stresses: Mechanisms of drought and mineral stress tolerance. Crop Science 40: 923-940.

Müller, J. 2003. Artificial infection by endophytes affects growth and mycorrhizal colonisation of Lolium perenne. Functional Plant Biology 30: 419424.

Omacini, M.; Eggers, T.; Bonkowski, M.; Gange, A.C.; Jones, T.H. 2006. Leaf endophytes affect mycorrhizal status and growth of co-infected and neighbouring plants. Functional Ecology 20: 226-232.

Parsons, A.J.; Rasmussen, S.; Xue, H.; Newman, J.A.; Anderson, C.B.; Cosgrove, G.P. 2004. Some 'high sugar grasses' don't like it hot. Proceedings of the New Zealand Grassland Association 66: 265-272.

Rasmussen, S.; Parsons, A.J.; Bassett, S. 2007. High nitrogen supply and carbohydrate content reduce fungal endophyte and alkaloid concentration in Lolium perenne. New Phytologist 173: 787-797.

Rasmussen, S.; Parsons, A.J.; Xue, H.; Fraser, K.; Newman, J.A. 2008a. Metabolic profiles of Lolium 
perenne are differentially affected by nitrogen supply, carbohydrate content, and fungal endophyte infection. Plant Physiology 146: 1440-1453.

Rasmussen, S.; Parsons, A.J.; Popay, A.; Xue, H.; Newman, J.A. 2008b. Plant-endophyte herbivore interactions: more than just alkaloids? Plant Signaling and Behavior 3: 1-4.

Smith, S.E.; Read, D.J. 1997. Mycorrhizal Symbiosis. Academic Press, San Diego, CA, USA. 243-246 pp.

Vicari, M.; Hatcher, P.E.; Ayres, P.G. 2002. Combined effect of foliar and mycorrhizal endophytes on an insect herbivore. Ecology 83: 2452-2464.

Yoneyama, K.; Xie, X.; Kusumoto, D.; Sekimoto, H.; Sugimoto, Y.; Takeuchi, Y.; Yoneyama, K. 2007. Nitrogen deficiency as well as phosphorus deficiency in sorghum promotes the production and exudation of 5-deoxystrigol, the host recognition signal for arbuscular mycorrhizal fungi and root parasites. Planta 227: 125-132. 